\title{
Clip Device
}

National Cancer Institute

\section{Source}

National Cancer Institute. Clip Device. NCI Thesaurus. Code C49873.

A small device designed to hold and attach items together. 